\title{
OUTER MEASURES AND ASSOCIATED LATTICE PROPERTIES
}

PETER M. GRASSI

\author{
Department of Mathematics \\ Hofstra University \\ Hempstead, NY 11550
}

(Received January 30, 1992 and in revised form May 18, 1992)

\begin{abstract}
Zero-one measure characterizations of lattice properties such as normality are extended to more general measures. For a given measure, we consider two associated "outer" measures and attempt to obtain the "outer"-measurable sets. We also seek necessary and sufficient conditions for the measure and outer measures to be equal on the lattice or its complement.
\end{abstract}

KEY WORDS AND PHRASES. Measure, lattice, outer-measure, measurable, normal, regular, almost countably compact, separates, semiseparates, support.

1991 AMS SUBJECT CLASSIFICATION CODES. 28C15, 28 A12.

\section{INTRODUCTION.}

Let $X$ be an arbitrary set and $\ell$ a lattice of subsets of $X . \quad \ell(\ell)$ denotes the algebra generated by $\ell$, and $M(\ell)$ those bounded and finitely additive measures on $\mathcal{\ell}(\ell) ; M_{R}(\ell)$ denotes those $\mu \in M(\ell)$ which are $\ell$-regular, while $M_{\sigma}(\ell)$ denotes those $\mu \in M(\ell)$ which are $\sigma$-smooth on $\ell$. Finally $I(\ell), I_{R}(\ell)$, and $I_{\sigma}(\ell)$ are the nontrivial zero-one elements of $M(\ell), M_{R}(\ell)$, and $M_{\sigma}(\ell)$ respectively.

Many well-known lattice properties can be completely characterized in terms of $I(\ell), I_{R}(\ell)$ or $I_{\sigma}(\ell)$, e.g., normal lattices, regular lattices, disjunctive lattices, etc. (see [4], [5], [3], [10], [2]). We begin by extending many of these results to $M(\ell), M_{R}(\ell)$, and $M_{\sigma}(\ell)$, especially in the case of a normal lattice (see Section 3).

In general, if $\nu$ is an arbitrary outer measure on the power set of $X$, it is very difficult to give a description of the $\nu$-measurable sets, or even to give nontrivial classes of sets which are $\nu$ measurable. In the case of $\mu$, a measure, and $\nu=\mu^{*}$ the induced outer measure, then, of course, classes of sets which are $\nu$-measurable are well-known. This is also the case in metric spaces with $\nu$ a metric outer measure. Here, we consider $\mu \in M(\ell)$ or $M_{\sigma}(\ell)$ and two associated "outer" measures $\nu=\mu^{\prime}$ and $\nu=\mu^{\prime \prime}$ and attempt to obtain the $\nu$-measurable sets. A full description can be given in case $\mu \in I(\ell)$ or $I_{\sigma}(\ell)$ (see Section 4), and we attempt to extend some of these results to the more general situation. We also seek necessary and sufficient conditions for various of the $\mu, \mu^{\prime}, \mu^{\mu}$ to be equal on $\mathcal{\ell}$ or $\ell^{\prime}$, the complementary lattice, under varying conditions on the measure and on $\mathcal{L}$.

Finally, in Section 5, we give lattice separating conditions between pairs of lattices $\ell_{1}$ and $\ell_{2}$ in terms of $\mu^{\prime}, \mu^{\prime \prime}$ or some other "outer" measure.

We begin by giving a brief review of the basic lattice and measure theoretic terminology and notation which will be used throughout the paper. This terminology will be consistent with standard usage (see e.g. [1], [6], [7], [8], [9], [11]). 


\section{BACKGROUND AND NOTATION.}

We shall let $\ell$ denote a lattice of subsets of a set $X$ and shall assume that the empty set and $X$ are in $\ell$. $\ell(\ell)$ denotes the algebra generated by $\ell$. If $\ell$ is closed under countable intersections then $\mathcal{L}$ is said to be a $\delta$-lattice. $\mathcal{L}$ is said to be normal if whenever $A, B \in \mathcal{L}$ such that $A \cap B=\emptyset$, there exist $C, D \in \ell$ such that $A \subset C^{\prime}, B \subset D^{\prime}$ and $C^{\prime} \cap D^{\prime}=0$. $\quad L$ is regular if for each $x \in X$ and $A \in \mathcal{L}$ such that $x \notin A$, there exist $B, C \in \mathcal{l}$ with $x \in B^{\prime}, A \subset C^{\prime}$ and $B^{\prime} \cap C^{\prime}=0 . \quad \ell$ is complement generated if for all $L \in \mathcal{L}, L=\bigcap_{\bigcap^{+}}^{+\infty} A_{i} \mathcal{L}$ is complement generated if for all $L \in \mathcal{L}, L=\bigcap^{+\infty} A_{2}^{\prime}, A_{i} \in \mathcal{L}$. $\mathcal{L}$ is countably paracompact if whenever $\left\{A_{i}\right\}$ is a decreasing sequence of lattice sets ${ }^{\prime}=1$ a decreasing sequence of $\ell^{\prime}$ sets $\left\{B_{i}^{\prime}\right\}$ such that $A_{i} \subset B_{i}^{\prime}$ for all $i$ and $\bigcap_{i=1}^{+\infty} B_{i}^{\prime \prime=}=\theta^{\prime}$. If $\ell_{1}$ and $\ell_{2}$ are lattices of subsets of $X$ and $\ell_{1} \subset \ell_{2}$, then $\ell_{1}$ separates $\ell_{2}$ if whener ${ }^{\prime} A, B \in \ell_{2}$ such that $A \cap B=0$, there exist $C, D \in \ell_{1}$ such that $A \subset C, B \subset D$, and $C \cap D=0 ; \ell_{1}$ semiseparates $\ell_{2}$ if whenever $A \in \ell_{1}$ and $B \in \ell_{2}$ such that $A \cap B=0$, there exists $C \in \ell_{1}$ such that $B \subset C$ and $A \cap C=\emptyset$. If $\ell_{1}$ separates $\ell_{2}$ then $\ell_{1}$ is normal if and only if $\ell_{2}$ is normal.

$M(\ell)$ denotes the set of all bounded and finitely additive measures defined on $\mathcal{l}(\ell)$. Without loss of generality, we assume that these measures are non-negative. A measure $\mu$ is $\sigma$-smooth on $\ell$ if $L_{i} \in \ell$ and $L_{i} \downarrow$ implies $\mu\left(L_{i}\right) \rightarrow 0 . \quad M^{\sigma}(\ell)$ will denote the set of all bounded and finitely additive measures which are $\sigma$-smooth, and hence countably additive, on $\mathcal{l}(\ell)$. If for all $A \in \mathcal{l}(\ell), \mu(A)=$ sup $\mu(L)$, where $L \subset A, L \in \mathcal{L}$, then $\mu$ is said to be $\ell$-regular. $M_{R}(\ell)$ denotes the subset of $M(\ell)$ consisting of all $\ell$-regular measures, and $M_{R}{ }^{\sigma}(\ell)$ that subset of $M_{R}(\ell)$ consisting of $\sigma$-smooth, $\ell$ regular measures, i.e., $M_{R}{ }^{\sigma}(\ell)=M_{R}(\ell) \cap M^{\sigma}(\ell)$. $M_{\sigma}(\ell)$ denotes those measures in $M(\ell)$ which are $\sigma$-smooth on $\ell$. $I(\ell)$ denotes the subset of $M(\ell)$ containing precisely the $0-1$ nontrivial measures; similarly, $I_{R}(\ell), I^{\sigma}(\ell), I_{R}{ }^{\sigma}(\ell)$ and $I_{\sigma}(\ell)$ denote those subsets of $M_{R}(\ell), M^{\sigma}(\ell), M_{R}{ }^{\sigma}(\ell)$ and $M_{\sigma}(\ell)$ respectively, which are in $I(\ell)$. We note that there is a one-to-one correspondence between prime filters on $\ell$ and measures in $I(\ell)$, and between $\ell$-ultrafilters and measures in $I_{R}(\ell)$. Furthermore, a prime filter on $\ell$ has the countable intersection property (i.e., the intersection of any countable number of prime filter set is nonempty) if and only if the corresponding measure is in $I_{\sigma}(\ell)$. If $\mu \in M(\mathcal{L}), S(\mu)$ denotes the support of $\mu$, i.e., $S(\mu)=\cap L$ such that $L \in \mathcal{L}$ and $\mu(L)=\mu(X)$. If $\mu, \nu \in M(\ell)$ we will write $\mu \leq \nu(\ell)$, or $\mu \leq \nu$ on $\ell$, whenever $\mu(L) \leq \nu(L)$ for all $L \in \ell$. One can show (c.f. [10]) that if $\mu \in M(\ell)$ then there exists a $\nu \in M_{R}(\ell)$ such that $\mu \leq \nu(\ell)$ and $\mu(X)=\nu(X)$; if $\ell$ is normal and $\mu \in I(\ell)$, then $\nu \in I_{R}(\ell)$ and $\nu$ is unique.

Similarly,

$$
I_{s}(\ell)=\left\{\mu \in I(\ell) \mid \text { if } L=\bigcap_{i=1}^{\infty} L_{i}, L, L_{i} \in \ell \text {, then } \mu(L)=\text { inf } \mu\left(L_{i}\right)\right\}
$$

$$
M_{s}(\mathcal{L})=\left\{\mu \in M(\ell) \mid \text { if } L=\bigcap_{i=1}^{\infty} L_{i}, L, L_{i} \in \mathcal{L} \text {, then } \mu(L)=\text { inf } \mu\left(L_{i}\right)\right\} .
$$

If $\ell$ is normal and complement generated then $\mu \in I_{s}(\ell)$ implies $\mu \in I_{R}{ }^{\sigma}(\ell)$ (c.f. [5]). Essentially the same proof shows that $\mu \in M_{s}(\ell)$ implies $\mu \in M_{R}{ }^{\sigma}(\ell)$. If $\ell$ is normal, $\mu \in I_{R}(\ell)$ and $\rho \in I_{R}\left(\ell^{\prime}\right)$, $\rho \leq \mu(\ell)$, then $\mu\left(L^{\prime}\right)=\sup \rho(A), A \subset L^{\prime}, A, L \in \ell$. If $\ell_{1} \subset \ell_{2}$ where $\ell_{1}$ separates $\ell_{2}$ and if $\mu \in I_{R}\left(\ell_{1}\right)$, $\nu \in I_{R}\left(\ell_{2}\right)$ where $\nu$ extends $\mu$, then $\nu$ is $\ell_{1}$-regular on $\ell_{2}^{\prime}$, and $\nu$ is unique; furthermore, if $\mu \in I_{R}{ }^{\sigma}\left(\ell_{1}\right)$ then $\nu \in I_{R}\left(\ell_{2}\right) \cap I_{\sigma}\left(\ell_{2}^{\prime}\right)$.

\section{EXTENSIONS OF SOME RESULTS TO MORE GENERAL MEASURES.}

It is interesting to note how results of Section 2 generalize and extend to measures which are not zero-one, i.e., to elements of $M(\ell), M_{R}(\ell)$ etc. We elaborate on a number of these below.

LEMMA 3.1. Let $\ell$ be normal, $\mu \in M(\ell), \mu \leq \nu(\ell)$ where $\nu \in M_{R}(\ell)$ and $\mu(X)=\nu(X)$. Then for $L \in \mathcal{L}, \nu\left(L^{\prime}\right)=\sup \mu(A), A \subset L^{\prime}, A \in \mathcal{L}$. 
PROOF. Let $\nu \in M_{R}(\mathcal{l})$. Then for $\varepsilon>0$ and $L \in \mathcal{L}$, there exists $L_{1} \in \mathcal{L}, L_{1} \subset L^{\prime}$ such that $\nu\left(L^{\prime}\right)-\nu\left(L_{1}\right)<\varepsilon$. Since $\mathcal{L}$ is normal, there exist $A, B \in \mathcal{L}$ such that $L_{1} \subset A^{\prime} \subset B \subset L^{\prime}$. Therefore $\mu(B) \geq \mu\left(A^{\prime}\right) \geq \nu\left(A^{\prime}\right) \geq \nu\left(L_{1}\right)$. Therefore, $\nu\left(L^{\prime}\right)-\mu(B)<\varepsilon$.

THEOREM 3.1. Suppose $\ell$ is normal, $\mu \in M(\ell), \nu_{1}, \nu_{2} \in M_{R}(\ell), \mu \leq \nu_{1}(\ell), \mu \leq \nu_{2}(\ell)$ and $\mu(X)=\nu_{1}(X)=\nu_{2}(X)$. Then $\nu_{1}=\nu_{2}$.

PROOF. It follows from Lemma 3.1 that $\nu_{1}\left(L^{\prime}\right)=\nu_{2}\left(L^{\prime}\right)$ for all $L \in \mathcal{L}$. Therefore, $\nu_{1}=\nu_{2}$.

Let $\mu \in M(\ell)$. Define $\rho(E)=\sup \mu\left(L^{\prime}\right), L^{\prime} \subset E, E \subset X$ and $L \in \mathcal{L}$. Our next result shows that the supports of $\mu$ and $\rho$ are equal if $\ell$ is regular. The definition of support is extended to $\rho$ in an obvious way.

THEOREM 3.2. If $\ell$ is regular then $S(\mu)=S(\rho)$.

PROOF. Since $\rho(L) \leq \mu(L)$ for all $L \in \mathcal{L}, S(\mu) \subset S(\rho)$.

Suppose there exists an $x \in S(\rho)$ and $x \notin S(\mu)$. If $\rho(L)=\rho(X)$, and $L \in \mathcal{L}$ then $x \in L$. But there exists $A \in \mathcal{L}$ such that $\mu(A)=\mu(X)$ and $x \notin A$. Since $\mathcal{l}$ is regular, there exist $L_{1}, L_{2} \in \mathcal{L}$ such that $x \in L_{1}^{\prime} \subset L_{2} \subset A^{\prime}$. Therefore $\mu\left(L_{2}^{\prime}\right) \geq \mu(A)=\mu(X)$. It follows that $\rho\left(L_{2}^{\prime}\right)=\rho(X)$. Therefore $\rho\left(L_{1}\right)=\rho(X)$ and hence $x \in L_{1}$, a contradiction.

DEFINITION. $\ell$ is almost countably compact (a.c.c.) if $\mu \in I_{R}\left(\ell^{\prime}\right)$ implies $\mu \in I_{\sigma}(\ell)$.

We now show that if $\ell$ is a.c.c. then its defining condition holds for general measures.

THEOREM 3.3. Suppose $\ell$ is a.c.c. Then $\mu \in M_{R}\left(\ell^{\prime}\right)$ implies $\mu \in M_{\sigma}(\ell)$.

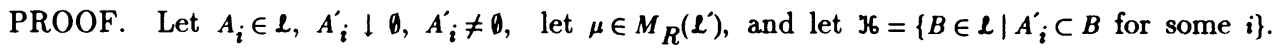
Now $\left\{A_{i}^{\prime}\right\}$ can be enlarged to an $\ell^{\prime}$ ultrafilter. Therefore, there exists $\nu \in I_{R}\left(\mathcal{L}^{\prime}\right)$ such that $\nu\left(A_{i}^{\prime}\right)=1$ for all $i$. Since $\mathcal{L}$ is a.c.c., $\nu \in I_{\sigma}(\mathcal{L})$. Thus 36 has the countable intersection property. Suppose $\ell_{i} \mid \boldsymbol{l}$,

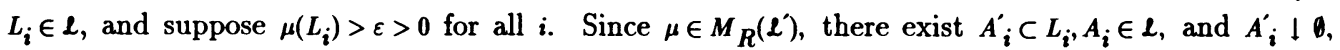
such that $\mu\left(A_{i}^{\prime}\right)>\varepsilon / 2$ for all $i$. Now $A_{i}^{\prime} \neq$ for any $i$ and $L_{i} \in \mathcal{J}$. Therefore $J_{6}$ does not have the countable intersection property, a contradiction.

THEOREM 3.4. Suppose $\ell_{1} \subset \ell_{2}$ where $\ell_{1}$ separates $\ell_{2}$. Let $\mu \in M_{R}\left(\ell_{1}\right), \nu \in M_{R}\left(\ell_{2}\right)$ and let $\nu$ extend $\mu$. Then the following are true:

a) $\quad \nu$ is $\ell_{1}$-regular on $\ell_{2}^{\prime}$.

b) If $\nu_{1} \in M_{R}\left(\ell_{2}\right)$ and $\nu_{1}$ extends $\mu$ then $\nu=\nu_{1}$.

c) $\quad \mu \in M_{R}{ }^{\sigma}\left(\ell_{1}\right)$ implies $\nu \in M_{R}\left(\ell_{2}\right) \cap M_{\sigma}\left(\ell_{2}^{\prime}\right)$.

d) $\quad \mu \in M_{R}{ }^{\sigma}\left(\ell_{1}\right)$ and $M_{\sigma}\left(\ell_{2}^{\prime}\right) \subset M_{\sigma}\left(\ell_{2}\right)$ implies $\nu \in M_{R}{ }^{\sigma}\left(\ell_{2}\right)$.

PROOF. a) Let $L_{2} \in \ell_{2}$. Since $v \in M_{R}\left(\ell_{2}\right)$, for any $\varepsilon>0$, there exists $A_{2} \in \ell_{2}$ such that $\nu\left(L_{2}^{\prime}\right)<\nu\left(A_{2}\right)+\varepsilon, A_{2} \subset L_{2}^{\prime}$. Since $\ell_{1}$ separates $\ell_{2}$, there exist $L_{1}, A_{1} \in \ell_{1}$ such that $L_{2} \subset L_{1}, A_{2} \subset A_{1}$, and $L_{1} \cap A_{1}=\emptyset$, i.e., $A_{2} \subset A_{1} \subset L_{1}^{\prime} \subset L_{2}^{\prime}$. Therefore, $\nu\left(L_{2}^{\prime}\right)<\nu\left(A_{2}\right)+\varepsilon<\nu\left(A_{1}\right)+\varepsilon$.

b) If $\nu$ and $\nu_{1} \in M_{R}\left(\ell_{2}\right)$ and are extensions of $\mu$, it follows from part a) that $\nu\left(L_{2}^{\prime}\right)=\nu_{1}\left(L_{2}^{\prime}\right)$ for all $L_{2} \in \ell_{2}$. Therefore $\nu=\nu_{1}$.

c) Let $\nu \in M_{R}\left(\ell_{2}\right)$ be an extension of $\mu \in M_{R}{ }^{\sigma}\left(\ell_{1}\right)$, let $A_{i}^{\prime} \downarrow \emptyset, A_{i} \in \ell_{2}$ and let $\varepsilon>0$ be given. Since $\nu$ is $\ell_{1}$-regular on $\ell_{2}^{\prime}$, there exist $B_{i} \subset A_{i}^{\prime}, B_{i} \downarrow \emptyset, B_{i} \in \ell_{1}$, such that $\nu\left(A_{i}^{\prime}\right)<\mu\left(B_{i}\right)+\varepsilon / 2$. Since $\mu \in M_{R}{ }^{\sigma}\left(\ell_{1}\right)$, there exists $B_{N} \in \ell_{1}$ such that $\mu\left(B_{N}\right)<\varepsilon / 2$. Thus $\nu\left(A_{N}^{\prime}\right)<\varepsilon$ and hence $\nu \in M_{\sigma}\left(\ell_{2}^{\prime}\right)$.

d) From c), $\nu \in M_{R}\left(\ell_{2}\right) \cap M_{\sigma}\left(\ell_{2}^{\prime}\right) \subset M_{R}\left(\ell_{2}\right) \cap M_{\sigma}\left(\ell_{2}\right)=M_{R}{ }^{\sigma}\left(\ell_{2}\right)$.

THEOREM 3.5. Suppose $\ell$ is normal. Let $\mu \in M_{\sigma}(\ell), \nu \in M_{R}(\ell), \mu \leq \nu(\ell)$ and $\mu(X)=\nu(X)$. Then $\nu \in M_{\sigma}\left(\boldsymbol{\ell}^{\prime}\right)$.

PROOF. Let $L_{i}^{\prime} \downarrow \ell, L_{i} \in \ell$ for all $i$ and let $\varepsilon>0$.

Since $\nu \in M_{R}(\mathcal{L})$, there exist $A_{i} \in \mathcal{L}$ such that $A_{i} \downarrow \emptyset, A_{i} \subset L_{i}^{\prime}$ and $\nu\left(L_{i}^{\prime}\right)<\nu\left(A_{i}\right)+\varepsilon / 2$. Since $\mathcal{L}$ is normal, there exist $B_{i}, C_{i} \in \mathcal{L}$ such that $A_{i} \subset B_{i}^{\prime} \subset C_{i} \subset L_{i}^{\prime}$ for all $i$, where $B_{i}^{\prime} \downarrow \emptyset$ and $C_{i} \downarrow \theta$. Therefore, $\nu\left(L_{i}^{\prime}\right)<\nu\left(B_{i}^{\prime}\right)+\varepsilon / 2 \leq \mu\left(B_{i}^{\prime}\right)+\varepsilon / 2 \leq \leq \mu\left(C_{i}\right)+\varepsilon / 2$. 
Since $\mu \in M_{\sigma}(\ell)$, there exists $C_{N}$ such that $\mu\left(C_{N}\right)<\varepsilon / 2$. It follows that $\nu\left(L_{N}^{\prime}\right)<\varepsilon$.

4. ASSOCIATED OUTER MEASURES AND LATTICE PROPERTIES.

In this section we introduce the associated "outer" measures $\mu^{\prime}$ and $\mu^{\prime \prime}$ and compare the behavior of $\mu \in I(\ell)$ or $I_{\sigma}(\ell)$ with that of $\mu \in M(\ell)$ or $M_{\sigma}(\ell)$. We consider relationships between $\mu^{\prime}$ and $\mu$ under added lattice assumptions.

Let $\mu \in M(\mathcal{L})$ and let $E \subset X$. Define $\mu^{\prime}(E)=$ inf $\mu\left(L^{\prime}\right)$, where inf is taken over all $L^{\prime}$ sets such that $E \subset L^{\prime}, L \in \mathcal{L}$.

Let $\mu \in M_{\sigma}(\ell)$ and let $E \subset X$. Define $\mu^{\prime \prime}(E)=$ inf $\sum_{i=1}^{\infty} \mu\left(L_{i}^{\prime}\right)$, where inf is taken over all $L_{i}^{\prime}$ sets such that $E \subset \underset{i=1}{\bigcup} L_{i}^{\prime}, L_{i} \in \ell$.

It is evident that $\mu^{\prime}$ ' is a finitely subadditive "outer" measure, i.e., $\mu^{\prime}$ has the defining properties of an outer measure with the exception that it is only finitely subadditive. On the other hand, $\mu^{\sim}$ is an outer measure. For the following reason, we assume $\mu \in M_{\sigma}(\ell)$ when defining $\mu^{\mu}$. If $\mu^{\mu}$ were defined for all $\mu \in M(\ell)$ then in particular, if $\mu \in I(\ell)$ and $\mu \notin I_{\sigma}(\ell)$ then $\mu^{\prime \prime} \equiv 0$.

THEOREM 4.1. Let $\mu \in M_{\sigma}(l)$. Then

a) $\mu^{\prime \prime} \leq \mu^{\prime}$ everywhere

b) $\mu(X)=\mu(X)$

c) $\mu \leq \mu$ " on $\ell$.

PROOF. a) Clear.

b) Clearly $\mu^{\prime \prime}(X) \leq \mu(X)$. Let $\varepsilon>0$ be given. Then there exist $L_{i} \in \mathcal{L}$ such that $X=\bigcup_{i=1}^{\infty} L_{i}^{\prime}$, and $\mu^{\prime \prime}(X)+\varepsilon>\sum_{i=1}^{\infty} \mu\left(L_{i}^{\prime}\right)$. Let $A_{N}^{\prime}=\bigcup_{=1}^{N} L_{i}^{\prime}$. Then, $\sum_{i=1}^{\infty} \mu\left(L_{i}^{\prime}\right) \geq \sum_{i=1}^{N} \mu\left(L_{i}^{\prime}\right) \geq \mu\left(A_{N}^{\prime}\right)=\mu(X)-\mu\left(A_{N}\right)$. Since $A_{N} \downarrow \theta$, it follows that $\mu^{\prime \prime}(X)+\varepsilon>\mu(X)$.

c) Let $L \in \mathcal{L}$. Then $\mu^{\prime \prime}(L) \geq \mu^{\prime \prime}(X)-\mu^{\prime \prime}\left(L^{\prime}\right)=\mu(X)-\mu^{\prime \prime}\left(L^{\prime}\right) \geq \mu(X)-\mu\left(L^{\prime}\right)=\mu(L)$.

REMARK. Let $E \subset X$ and let $\mu^{*}(E)=$ inf $\mu(A), E \subset A, A \in \mathcal{L}(\ell)$.

We note that under the assumptions of Theorem 3.4, $\nu=\mu^{*}=\mu^{\prime}$ on $\ell_{2}$.

The following theorem concerning supports is a generalization of a result in [5]. We omit the proof since it is essentially the same proof given there (c.f. Theorem 4.10 [5]) and note a corollary pertaining to $\mu$.

THEOREM 4.2. Let $\mu \in M(\ell)$ where $\ell$ is regular. Let $\tau$ be a monotone set function defined on any collection of sets containing $\mathcal{\ell}(\mathcal{\ell})$, where $\tau \geq 0, \mu \leq \tau$ on $\mathcal{L}, \tau \leq \mu$ on $\ell^{\prime}$, and $\tau(X)=\mu(X)$. Then $S(\tau)=S(\mu)$.

COROLLARY 4.1. Let $\mu \in M(\ell)$, and let $\ell$ be regular. Then $S\left(\mu^{\prime}\right)=S(\mu)$.

Let $\sigma_{\mu^{\prime}}$ denote the collection of $\mu^{\prime}$-measurable sets, and let $\sigma_{\mu^{\prime \prime}}$ denote the collection of $\mu^{\prime \prime}$ measurable sets. Theorem 4.3 presents a classification of these sets for $0-1$ measures.

THEOREM 4.3. a) Let $\mu \in I(\ell)$.

Then $g_{\mu^{\prime}}=\left\{E \subset X \mid E \supset L\right.$ or $\left.E^{\prime} \supset L, L \in \ell, \mu(L)=1\right\}$.

b) Let $\mu \in I_{\sigma}(\mathcal{l})$. Then $\sigma_{\mu^{\mu}}=\left\{E \subset X \mid E \supset \bigcap_{i=1}^{\infty} L_{i}\right.$ or $E^{\prime} \supset \bigcap_{i=1}^{\infty} L_{i}, \mu\left(L_{i}\right)=1, L_{i} \in \mathcal{L}$ for all $\left.i\right\}$.

PROOF. a) Let $E \subset X$, and $L \in \ell$.

If $L \subset E$ and $\mu(L)=1$ then $\mu^{\prime}(E)=1$ and $\mu^{\prime}\left(E^{\prime}\right)=0$.

If $L \subset E^{\prime}$ and $\mu(L)=1$ then $\mu^{\prime}\left(E^{\prime}\right)=1$ and $\mu^{\prime}(E)=0$.

In either case, $E \in \mathcal{T}_{\mu}$ "

Conversely, suppose $E \in \mathcal{J}_{\mu^{\prime \prime}}$ Then $\mu^{\prime}(E)+\mu^{\prime}\left(E^{\prime}\right)=1$.

If $\mu^{\prime}(E)=0$ then there exists $L \in \mathcal{L}$ such that $E \subset L^{\prime}$ and $\mu\left(L^{\prime}\right)=0$. Thus $\mu(L)=1$.

If $\mu^{\prime}\left(E^{\prime}\right)=0$ then there exists $L \in \mathcal{\ell}$ such that $L \subset E$ and $\mu(L)=1$.

b) Let $E \subset X, L_{i} \in \mathcal{L}, E \supset \bigcap_{i=1}^{\infty} L_{i}$ and $\mu\left(L_{i}\right)=1$ for all $i$. 


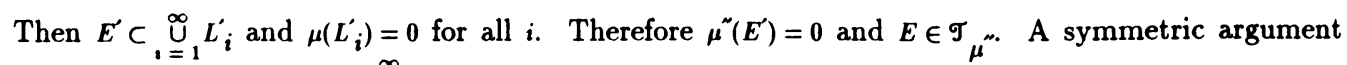
proves that $E \in \mathcal{T}_{\mu^{\prime \prime}}$ if $E^{\prime} \supset \bigcap_{i=1}^{\infty} L_{i}$.

Conversely, let $E \subset X$ and suppose $\mu^{\prime \prime}(A)=\mu^{\prime \prime}(A \cap E)+\mu^{\prime \prime}\left(A \cap E^{\prime}\right)$ for all $A \subset X$. In particular, $1=\mu^{\prime \prime}(X)=\mu^{\prime \prime}(E)+\mu^{\prime \prime}\left(E^{\prime}\right)$. If $\mu^{\prime \prime}(E)=0$ then there exist $L_{i} \in \mathcal{\ell}$ such that $E \subset, \bigcup_{=1}^{\infty} L_{i}^{\prime}$ and $\mu\left(L_{i}\right)=1$ for all i. Similarly, $\mu^{\prime \prime}\left(E^{\prime}\right)=0$ implies $E \supset \bigcap_{i=1}^{\infty} L_{i}$ and $\mu\left(L_{i}\right)=1$ for all $i$.

The sets $I_{s}(\mathcal{L})$ and $M_{s}(\mathcal{L})$ provide a framework from which many of the remaining theorems of this section rely, particularly with respect to results concerning $\mu^{\prime \prime}$ and the $\mu^{\prime \prime}$-measurable sets.

REMARK. If $\mu \in M(\ell)$ then $E \in \sigma_{\mu^{\prime}}$ iff $\mu^{\prime}\left(A^{\prime}\right) \geq \mu^{\prime}\left(A^{\prime} \cap E\right)+\mu^{\prime}\left(A^{\prime} \cap E^{\prime}\right)$ for all $A^{\prime} \in \mathcal{L}^{\prime}$.

LEMMA 4.1. Let $\mu \in M(\ell)$ and let $E \subset X$. Then $E \in \mathcal{T}_{\mu^{\prime}}$ if and only if $\mu^{\prime}(E)=\sup \mu(L)$ where $L \subset E$ and $L \in \mathcal{L}$.

PROOF. Suppose $\mu^{\prime}(E)=\sup \mu(L)$. Let $\varepsilon>0$ be given. Then there exists $L \in \mathcal{L}$ such that $L \subset E$ and $\mu^{\prime}(E)-\mu(L)<\varepsilon / 2$. Similarly, by definition of $\mu^{\prime}$, there exists $B^{\prime} \supset E$ such that $B \in \mathcal{L}$ and $\mu\left(B^{\prime}\right)-\mu^{\prime}(E)<\varepsilon / 2$. Therefore, $\mu\left(B^{\prime}\right)-\mu(L)<\varepsilon$ and $L \subset E \subset B^{\prime}$. Let $A^{\prime} \in \mathcal{L}^{\prime}$. Now $\mu^{\prime}\left(A^{\prime} \cap E\right) \leq \mu\left(A^{\prime} \cap B^{\prime}\right)=$ $\mu\left(A^{\prime}\right)+\mu\left(B^{\prime}\right)-\mu\left(A^{\prime} \cup B^{\prime}\right)<\mu\left(A^{\prime}\right)+\mu(L)+\varepsilon-\mu\left(A^{\prime} \cup L\right)=\mu\left(A^{\prime} \cap L\right)+\varepsilon$. Therefore, $\mu\left(A^{\prime} \cap L\right)>\mu^{\prime}\left(A^{\prime} \cap E\right)-\varepsilon$. It follows that, $\mu\left(A^{\prime}\right)=\mu\left(A^{\prime} \cap L\right)+\mu\left(A^{\prime} \cap L^{\prime}\right) \geq \mu\left(A^{\prime} \cap L\right)+\mu^{\prime}\left(A^{\prime} \cap E^{\prime}\right)>\mu^{\prime}\left(A^{\prime} \cap E\right)-\varepsilon+\mu^{\prime}\left(A^{\prime} \cap E^{\prime}\right)$ and hence $E \in \mathcal{T}_{\mu^{\prime \prime}}$ Conversely, suppose $E \in \mathcal{T}_{\mu^{\prime \prime}}$ Then $\mu^{\prime}\left(E^{\prime}\right)=\mu(X)-\mu^{\prime}(E)$. Also, if $\varepsilon>0$, there exists $L \in \mathcal{L}$ such that $L^{\prime} \supset E^{\prime}$ and $\mu\left(L^{\prime}\right)-\mu^{\prime}\left(E^{\prime}\right)<\varepsilon$. Therefore, $\mu^{\prime}(E)-\mu(L)<\varepsilon$.

THEOREM 4.4. a) Let $\mu \in M(\ell)$. Then $\sigma_{\mu^{\prime}} \cap \mathcal{L}=\left\{L \in \mathcal{L} \mid \mu^{\prime}(L)=\mu(L)\right\}$.

b) Let $\mu \in I_{\sigma}(\ell)$. Then $\sigma_{\mu}{ }^{\mu} \cap \ell=\left\{L \in \ell \mid \mu^{\mu}(L)=\mu(L)\right\}$ iff $\mu \in I_{\delta}(\ell)$.

PROOF. a) Clearly follows form Lemma 4.1 .

b) Let $\ell_{\mu^{\mu}}=\left\{L \in \mathcal{L} \mid \mu^{\mu}(L)=\mu(L)\right\}$. Suppose $\sigma_{\mu^{\mu}} \cap \mathcal{L}=\mathcal{L}_{\mu^{\mu}}$. Let $L_{i} \downarrow L$, where $L_{i} L \in \mathcal{L}$. Suppose $\mu\left(L_{i}\right)=1$ for all $i$. Then $L \in \sigma_{\mu} \cap \cap \mathcal{L}$. If $\mu(L)=0$ then $\mu^{\mu}(L)=0$. Therefore there exist $A_{i} \in \mathcal{L}, A_{i} \downarrow, L \subset, \bigcup_{i=1}^{\infty} A_{i}^{\prime}$ and $\mu\left(A_{i}^{\prime}\right)=0$ for all $i$. Therefore, $\left(L_{i} \cap A_{i}\right) \downarrow 0$. But $\mu\left(L_{i} \cap A_{i}\right)=1$ for all $i, \mathrm{a}$ contradiction since $\mu \in I_{\sigma}(\mathcal{\ell})$. Conversely, suppose $\mu \in I_{s}(\ell)$ and $L \in \mathcal{T}_{\mu^{\mu}} \cap \mathcal{L}$. Let $L, L_{i} \in \mathcal{\ell}$.

CASE 1: $\prod_{i=1}^{\infty} L_{i} \subset L$ and $\mu\left(L_{i}\right)=1$ for all $i$.

Then $\bigcap_{i=1}^{\infty}\left(L \cap L_{i}\right)=L$ and we may assume $\left(L \cap L_{i}\right) \mid$.

Since $\mu \in I_{s}(\ell), \mu(L)=1$. Therefore $\mu^{\mu}(L)=1$.

CASE 2: $\bigcap_{i=1}^{\infty} L_{i} \subset L^{\prime}$ and $\mu\left(L_{i}\right)=1$ for all $i$.

Then $\mu\left(L_{i}^{\prime}\right)=0$ for all $i$ and $L \subset \bigcup_{i=1}^{\infty} L_{i}^{\prime}$. Therefore $\mu^{\prime \prime}(L)=0$ and hence $\mu(L)=0$.

Thus $\sigma_{\mu^{\mu}} \cap \mathcal{L} \subset \mathcal{L}_{\mu}$. But clearly, $\mathcal{T}_{\mu^{\mu}} \cap \mathcal{L} \supset \mathcal{L}_{\mu}{ }_{\mu}$.

THEOREM 4.5. Suppose $\mu \in M(\ell)$. Then $\ell \subset \sigma_{\mu^{\prime}}$ iff $\mu \in M_{R}(\ell)$.

PROOF. Clear.

We next investigate conditions which guarantee that $\mu^{\prime}$ and $\mu^{\mu}$, or $\sigma_{\mu^{\prime}}$ and $\sigma_{\mu^{\prime \prime}}$ are equal. Our first result concerns the equality of these outer measures on $\ell^{\prime}$.

THEOREM 4.6. Let $\mu \in M_{\sigma}(\ell)$. If $\mu \in M_{g}(\ell)$ then $\mu^{\prime}=\mu^{\prime \prime}$ on $\ell^{\prime}$.

PROOF. We know that $\mu^{\prime \prime} \leq \mu^{\prime}$ on $\ell^{\prime}$. Let $\varepsilon>0$ be given and let $L \in \ell$. Then there exist $L_{i} \in \mathcal{L}$ such that $L^{\prime} \subset \bigcup_{i=1}^{\infty} L_{i}^{\prime}$ and $\mu^{\prime \prime}\left(L^{\prime}\right)+\varepsilon / 2>\sum_{i=1}^{\infty} \mu\left(L_{i}^{\prime}\right)$. (We assume $L_{i}^{\prime} \dagger$.) Therefore, $L_{=} \prod_{i=1}^{\infty}\left(L_{i} \cup L\right)$ and $\left(L_{i} \cup L\right) \downarrow L$. Since $\mu \in M_{s}(\ell)$, there exists $L_{N}$ such that $\mu\left(L_{N} \cup L\right)-\mu(L)<\varepsilon / 2$ for some $N$, or equivalently, $\mu\left(L_{N}^{\prime} \cap L^{\prime}\right)>\mu\left(L^{\prime}\right)-\varepsilon / 2$.

Therefore,

$$
\mu^{\prime \prime}\left(L^{\prime}\right)+\varepsilon / 2>\sum_{i=1}^{\infty} \mu\left(L_{i}^{\prime}\right) \geq \mu\left(L_{N}^{\prime}\right) \geq \mu\left(L_{N}^{\prime} \cap L^{\prime}\right)>\mu\left(L^{\prime}\right)-\varepsilon / 2
$$

from which it follows that $\mu^{\prime \prime}\left(L^{\prime}\right)=\mu\left(L^{\prime}\right)=\mu^{\prime}\left(L^{\prime}\right)$. 
THEOREM 4.7. Let $\mu \in M_{\sigma}(\ell)$ and let $\ell$ be normal. Suppose either of the following two conditions is true:

i) $\mathcal{\ell}$ is countably paracompact.

ii) $\mathcal{L}$ is a $\delta$-lattice.

Then $\mu^{\prime}=\mu^{\prime \prime}$ on $\ell$.

PROOF. i) Since $\ell$ is normal and countably paracompact, there exists $v \in M_{R}{ }^{\sigma}(\ell)$ such that $\mu \leq \nu$ on $\ell$ and $\mu(X)=\nu(X)$. (c.f. [11]) Therefore, on $\ell, \nu=\nu^{\prime}=\nu^{\prime \prime} \leq \mu^{\prime \prime} \leq \mu^{\prime}$. Also, since $\ell$ is normal, $\nu^{\prime}=\mu^{\prime}$ on $\boldsymbol{\ell}$.

ii) Let $\varepsilon>0$ be given and let $L \in \mathcal{L}$. There exist $L_{i} \downarrow, L \subset \underset{,=1}{\bigcup} L_{i}^{\prime}$ and $\mu^{\prime}(L)+\varepsilon>, \sum_{=1}^{\infty} \mu\left(L_{i}^{\prime}\right)$. Since $\ell$ is $\delta$, if $A^{\prime}=\bigcup_{=1}^{\infty} L_{i}^{\prime}$ then $A \in \mathcal{l}$. Since $\mathcal{l}$ is normal, there exist $B, C \in \mathcal{\ell}$ such that $L \subset B^{\prime} \subset C \subset A^{\prime}$. Therefore,

$$
\mu^{\prime}(L) \leq \mu\left(B^{\prime}\right) \leq \mu(C) \leq \mu^{\prime \prime}(C) \leq \mu^{\prime \prime}\left(A^{\prime}\right) \leq,{ }_{1}^{\infty} \mu_{1}^{\infty} \mu\left(L_{i}^{\prime}\right)<\mu^{\prime \prime}(L)+\varepsilon .
$$

THEOREM 4.8. Suppose $\ell$ is $\delta$ and $\mu \in M_{s}(\ell)$. Then

a) $\mu\left(\bigcup_{i=1}^{\infty} L_{i}^{\prime}\right) \leq \sum_{i=1}^{\infty} \mu\left(L_{i}^{\prime}\right), L_{i} \in \ell$.

b) $\mu^{\prime}=\mu^{\prime \prime}$ everywhere

c) $\sigma_{\mu^{\prime}}=\sigma_{\mu}{ }_{\mu}$.

The following corollary follows immediately from Theorem 4.5 and Theorem $4.8 \mathrm{c}$ ).

COROLLARY 4.2. Suppose $\ell$ is $\delta, \mu \in M_{s}(\ell)$ and $\ell \subset \mathcal{J}_{\mu}$. Then $\mu \in M_{R^{\prime}}(\ell)$.

The following theorem shows that set inclusion of $\mu$-measurable sets is preserved under inequalities with respect to the lattice $\ell$.

THEOREM 4.9. Let $\mu, \nu \in M(\ell), \mu \leq \nu$ on $\mathcal{L}$ and $\mu(X)=\nu(X)$. Then $\sigma_{\mu^{\prime}} \subset \sigma_{\nu^{\prime}}$

PROOF. $E \in \mathcal{G}_{\mu^{\prime}}$ implies $\mu(E)=\sup \mu(L) \leq \sup \nu(L) \leq \nu^{\prime}(E), L \subset E, L \in \mathcal{\ell}$. But since $\mu \leq \nu$ on $\ell$, $\nu \leq \mu$ on $\ell^{\prime}$ and hence $\nu^{\prime}(E) \leq \mu^{\prime}(E)$.

We next note some extensions of some results which are known for zero-one measures that require the notion of a regular outer measure. We begin by defining this concept and list some consequences.

Let $\nu$ be a finitely subadditive outer measure. Then $\nu$ is regular if for every $G \subset X$, there exists $E \in \mathscr{T}_{\nu}$ such that $G \subset E$ and $\nu(G)=\nu(E)$.

The following properties are noted for completeness:

i) Let $\nu$ be a regular outer measure. If $E_{i} \uparrow, E_{i} \subset X$, then $\nu\left(\lim E_{i}\right)=\lim \nu\left(E_{i}\right)$.

ii) Let $\nu$ be a regular, finitely subadditive, outer measure. Then $E \in \mathcal{T}_{\nu}$ iff $\nu(X)=\nu(E)+\nu\left(E^{\prime}\right)$.

REMARK. Clearly, $i)$ is not true if $\nu$ is a finitely subadditive outer measure. For example, let $\mu \in I(\mathcal{L})-I_{\sigma}(\mathcal{l})$. Then there exist $L_{i} \in \mathcal{L}$ such that $L_{i} \downarrow \emptyset$ and $\mu\left(L_{i}\right)=1$ for all $i$. Therefore, $\mu^{\prime}(X)=\mu(X)=1$ but $\mu^{\prime}\left(L_{i}\right)=0$ for all $i$.

We now show that the converse of Theorem 4.6 is valid when $\mu^{\mu}$ is regular.

THEOREM 4.10. Let $\mu \in M_{\sigma}(\ell)$. If $\mu^{\prime}=\mu^{\prime \prime}$ on $\ell^{\prime}$ and if $\mu^{\prime}$ is regular then $\mu \in M_{8}(\ell)$.

PROOF. Suppose $\mu^{\prime}=\mu^{\nu}$ on $\ell^{\prime}$. Let $L_{i} \mid L, L_{i}, L \in \ell$. Assume there exists $\varepsilon>0$ such that $\mu(L)+\varepsilon<\mu\left(L_{i}\right)$ for all $i$. Then $\mu\left(L^{\prime}\right)-\varepsilon>\mu^{\prime \prime}\left(L_{i}^{\prime}\right)$ for all $i$ and hence $\mu\left(L^{\prime}\right)-\varepsilon \geq \lim \mu^{\prime \prime}\left(L_{i}^{\prime}\right)$ $=\mu^{\prime \prime}\left(\lim L_{i}^{\prime}\right)=\mu^{\prime \prime}\left(L^{\prime}\right)=\mu\left(L^{\prime}\right)$, a contradiction.

We end this section with some further consequences of regular outer measures which are stated without proof in the following theorem.

THEOREM 4.11. Let $\mu \in M_{\sigma}(\mathcal{l})$ and let $\mu^{\prime \prime}$ be regular. Then the following hold:

a) $\sigma_{\mu}, \subset \sigma_{\mu}{ }^{*}$.

b) If $\mu=\mu^{\mu}$ on $\ell$ then $\mu \in M^{\sigma}(\ell)$. 
Clearly, if $\mu \in I(\ell)$ then $\mu^{\prime}$ is regular. Similarly, if $\mu \in I_{\sigma}(\ell)$ then $\mu^{\sim}$ is regular. Therefore, the consequences of Theorems 4.10 and 4.11 are valid if $\mu \in I_{\sigma}(\ell)$. It is interesting to note that no explicit use of the regular assumption of outer measures is required in proving this case.

\section{ASSOCIATED OUTER MEASURES AND LATTICE SEPARATION.}

In this section we investigate the effect of further lattice assumptions on various measures and for this purpose introduce the class of measures $M_{W}(\ell)$. Also, we investigate the effect of assumptions concerning "outer" measures on lattice separation properties.

For $E \subset X$ define $\tilde{\mu}(E)=$ inf $\mu(L)$ where $E \subset L, L \in \mathcal{L}$. We note that $\tilde{\mu}$ is a finitely subadditive outer measure.

LEMMA 5.1. Let $\ell_{1} \subset \ell_{2}$ and suppose $\ell_{1}$ semiseparates $\ell_{2}$. If $\mu \in M\left(\ell_{1}\right)$ then $\mu^{\prime} \geq \tilde{\mu}$ on $\ell_{2}$.

PROOF. Let $\mu \in M\left(\ell_{1}\right), L_{2} \in \ell_{2}$ and $\varepsilon>0$. Then there exists $L_{1}^{\prime} \supset L_{2}, L_{1} \in \ell_{1}$ such that $\mu^{\prime}\left(L_{2}\right)>\mu\left(L_{1}^{\prime}\right)-\varepsilon$. If $\ell_{1}$ semiseparates $\ell_{2}$, there exists $A_{1} \in \ell_{1}$ such that $L_{2} \subset A_{1} \subset L_{1}^{\prime}$.

Thus,

$$
\mu^{\prime}\left(L_{2}\right)>\mu\left(L_{1}^{\prime}\right)-\varepsilon \geq \mu\left(A_{1}\right)-\varepsilon \geq \tilde{\mu}\left(L_{2}\right)-\varepsilon
$$

Therefore $\mu^{\prime} \geq \tilde{\mu}$ on $\ell_{2}$.

LEMMA 5.2. Let $\ell_{1} \subset \ell_{2}$. If $\mu \in M_{\left.R^{(} \ell_{1}\right)}$ then $\tilde{\mu} \geq \mu^{\prime}$ on $\ell_{2}$.

PROOF. Let $\varepsilon>0$ be given and let $L_{2} \in \ell_{2}$. Then there exists $L_{1} \in \ell_{1}$ such that $L_{1} \supset L_{2}$ and $\tilde{\mu}\left(L_{2}\right)>\mu\left(L_{1}\right)-\varepsilon$. Since $\mu \in M_{R}\left(\ell_{1}\right), \mu=\mu^{\prime}$ on $\ell_{1}$. Thus, $\tilde{\mu}\left(L_{2}\right)>\mu\left(L_{1}\right)-\varepsilon=\mu^{\prime}\left(L_{1}\right)-\varepsilon \geq \mu^{\prime}\left(L_{2}\right)-\varepsilon$. Therefore, $\tilde{\mu} \leq \mu^{\prime}$ on $\ell_{2}$.

Combining the results from Lemmas 5.1 and 5.2 we have,

THEOREM 5.1. Let $\ell_{1} \subset \ell_{2}$ and suppose $\ell_{1}$ semiseparates $\ell_{2}$. If $\mu \in M_{R}\left(\ell_{1}\right)$ then $\mu^{\prime}=\tilde{\mu}$ on $\ell_{2}$.

The following theorem gives conditions which preserve inequalities of measures when extended to super-lattices.

THEOREM 5.2. Suppose $\ell_{1} \subset \ell_{2}$ and $\ell_{1}$ separates $\ell_{2}$. Let $\mu \leq \nu\left(\ell_{1}\right)$ where $\mu \in M\left(\ell_{1}\right), \nu \in M_{R}\left(\ell_{1}\right)$, and let $\tau$ and $\lambda$ be extensions of $\mu$ and $\nu$ to $M\left(\ell_{2}\right)$ and $M_{R}\left(\ell_{2}\right)$ respectively. Then $\tau \leq \lambda\left(\ell_{2}\right)$.

PROOF. Suppose there exists $L_{2} \in \ell_{2}$ such that $\tau\left(L_{2}\right)>\lambda\left(L_{2}\right)$. Let $\varepsilon>0$ and let $\tau\left(L_{2}\right)-\lambda\left(L_{2}\right)>\varepsilon$. Since $\lambda \in M_{R}\left(\ell_{2}\right)$, there exists $A_{2}^{\prime} \supset L_{2}, A_{2} \in \mathcal{L}_{2}$ such that $\lambda\left(L_{2}\right)+\varepsilon>\lambda\left(A_{2}^{\prime}\right)$. Since $\ell_{1}$ separates $\ell_{2}$, there exist $L_{1}, A_{1} \in \ell_{1}$ such that $L_{2} \subset L_{1} \subset A_{1}^{\prime} \subset A_{2}^{\prime}$. Therefore,

$$
\mu\left(L_{1}\right)=\tau\left(L_{1}\right) \geq \tau\left(L_{2}\right)>\lambda\left(L_{2}\right)+\varepsilon>\lambda\left(A_{2}^{\prime}\right) \geq \lambda\left(L_{1}\right)=\nu\left(L_{1}\right),
$$

a contradiction.

We now show that semiseparation is a sufficient condition to preserve equality of the outer measures $\mu^{\prime}, \mu^{\prime \prime}$ on super-lattices when the given lattice is $\delta$.

THEOREM 5.3. Let $\ell_{1} \subset \ell_{2}$ where $\ell_{1}$ is $\delta, \ell_{1}$ semiseparates $\ell_{2}$, and let $\mu \in M_{\sigma}\left(\ell_{1}\right)$. If $\mu^{\prime}=\mu$ " on $\ell_{1}$ then $\mu^{\prime}=\mu^{\prime \prime}$ on $\ell_{2}$.

PROOF. If $\varepsilon>0$ and $L_{2} \in \ell_{2}$, there exist $L_{i} \in \ell_{1}$ such that $\stackrel{\bigcup \bigcup}{=1}_{1}^{\infty} L_{i}^{\prime} \supset L_{2}$ and $\mu^{\sim}\left(L_{2}\right)+\varepsilon>, \sum_{i=1}^{\infty} \mu\left(L_{1}^{\prime}\right)$. Since $\ell_{1}$ is $\delta, A_{1}^{\prime}=\bigcup_{i=1}^{\infty} L_{i}^{\prime}, A_{1} \in \ell_{1}$.

If $\ell_{1}$ semiseparates $\ell_{2}$ there exists $B_{1}$ in $\ell_{1}$ such that $L_{2} \subset B_{1} \subset A_{1}^{\prime}$. Now,

$$
\mu^{\prime}\left(L_{2}\right) \leq \mu^{\prime}\left(B_{1}\right)=\mu^{\prime \prime}\left(B_{1}\right) \leq \mu^{\prime \prime}\left(A_{1}^{\prime}\right) \leq \sum_{i=1}^{\infty} \mu\left(L_{i}^{\prime}\right)<\mu^{\prime \prime}\left(L_{2}\right)+\varepsilon .
$$

Therefore, $\mu^{\prime} \leq \mu^{\prime \prime}$ on $\ell_{2}$. Since $\mu^{\prime} \geq \mu^{\prime \prime}$ everywhere, the conclusion follows.

Let $M_{W}(\mathcal{L})=\left\{\mu \in M(\ell) \mid \mu\left(L^{\prime}\right)=\sup \mu^{\prime}(A), L, A \in \mathcal{L}\right.$ and $\left.A \subset L^{\prime}\right\}$.

Clearly, $M_{\left.R^{(}\right) \subset M_{W}}(\ell)$. We now prove that $M_{R}(\ell)=M_{W}(\ell)$ when $\ell$ is normal. 
THEOREM 5.4. Suppose $\ell$ is normal. Then $\mu \in M_{\left.W^{(}\right)}$implies that $\mu \in M_{R}(\boldsymbol{l})$.

PROOF. Let $\varepsilon>0$ and $L \in \mathcal{L}$. Since $\mu \in M_{W^{(\ell)}}$, there exists $L_{1} \in \mathcal{L}$ such that $L_{1} \subset L^{\prime}$ and $\mu\left(L^{\prime}\right)-\mu^{\prime}\left(L_{1}\right)<\varepsilon$. Since $\mathcal{l}$ is normal, there exist $A, B \in \mathcal{l}$ such that $L_{1} \subset A^{\prime} \subset B \subset L^{\prime}$.

Therefore, $\mu^{\prime}\left(L_{1}\right) \leq \mu^{\prime}\left(A^{\prime}\right)=\mu\left(A^{\prime}\right) \leq \mu(B) \leq \mu^{\prime}(B) \leq \mu\left(L^{\prime}\right)$ and hence, $\mu\left(L^{\prime}\right)-\mu(B)<\varepsilon$. It follows that $\mu \in M_{R}(\ell)$.

The following example shows that the converse of Theorem 5.4 is not true.

EXAMPLE 5.1. Let $A, B \subset X, A \cup B \neq X$ and $A \cap B=\emptyset$. Let $\ell=\{\emptyset, A, B, A \cup B, X\}$. Clearly $\ell$ is not normal, but $M_{W}(\ell)=M_{R}(\ell)$.

THEOREM 5.5. Let $\ell_{1} \subset \ell_{2}$ and let $\ell_{1}$ semiseparate $\ell_{2}$. If $\nu \in M_{W}\left(\ell_{2}\right)$ and if $\mu$ is the restriction of $\nu$ to $\Lambda\left(\ell_{1}\right)$ then $\mu \in M_{W}\left(\ell_{1}\right)$.

PROOF. Let $\varepsilon>0$ and let $L_{1} \in \ell_{1}$. Then there exists $L_{2} \subset L_{1}^{\prime}$ such that $\mu\left(L_{1}^{\prime}\right)-\varepsilon<\nu^{\prime}\left(L_{2}\right)$. Since $\ell_{1}$ semiseparates $\ell_{2}$, there exists $A_{1} \in \ell_{1}$ such that $L_{2} \subset A_{1} \subset L_{1}^{\prime}$. Clearly, $\nu^{\prime}\left(L_{2}\right) \leq \mu^{\prime}\left(L_{2}\right) \leq \mu^{\prime}\left(A_{1}\right)$. Therefore, $\mu\left(L_{1}^{\prime}\right)-\varepsilon<\mu^{\prime}\left(A_{1}\right)$.

\section{REFERENCES}

1. ALEXANDROFF, A.D., Additive set functions in abstract spaces, Mat. Sb., (N.S.) 8, 50 (1940), 307-348.

2. CAMACHO, JR., J., Extensions of lattice regular measures with applications, J. Indian Math. Soc. 54 (1989), 233-244.

3. EID, G., On normal lattices and Wallman spaces, Inter. J. of Math. and Math. Sci. Vol. 13, No. 1 (1990), 31-38.

4. FROLIK, Z., Prime filters with the C.I.P., Comm. Math. Univ. Carolinae 13, 3 (1972), 553-573.

5. GRASSI, P., Measure characterizations and properties of normal and regular lattices, Inter. J. of Math. and Math. Sci. Vol. 14, No. 3 (1991), 385-392.

6. GRASSI, P., On subspaces of replete and measure replete spaces, Canad. Math. Bull. 1, 27 (1984), 58-64.

7. GRASSI, P., An adjoint map between regular measures and its applications, Jour. Ind. Math. Soc. 48 (1984), 19-31.

8. HUERTA, C., Notions of compactness on the lattice and on the point set in terms of measure, Ann. Sc. Math., Quebec, 13, 1 (1989), 49-52.

9. NÖBELING, G., Grundlagen der Analytischen Topologie, Springer-Verlag, Berlin, 1954.

10. SZETO, M., On maximal measures with respect to a lattice, Measure Theory and its Applications, 1980, Northern Illinois University, 277-282.

11. SZETO, M., Measure repleteness and mapping preservations, Jour. Ind. Math. Soc. 43 (1979), 35-52. 


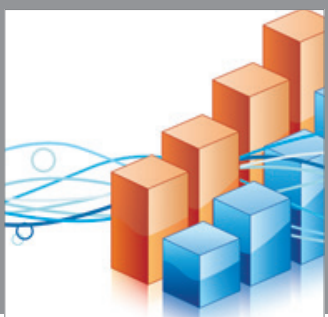

Advances in

Operations Research

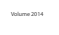

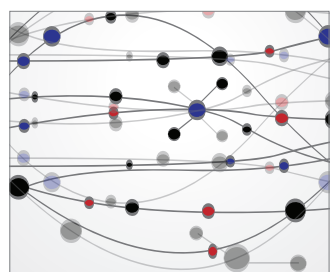

\section{The Scientific} World Journal
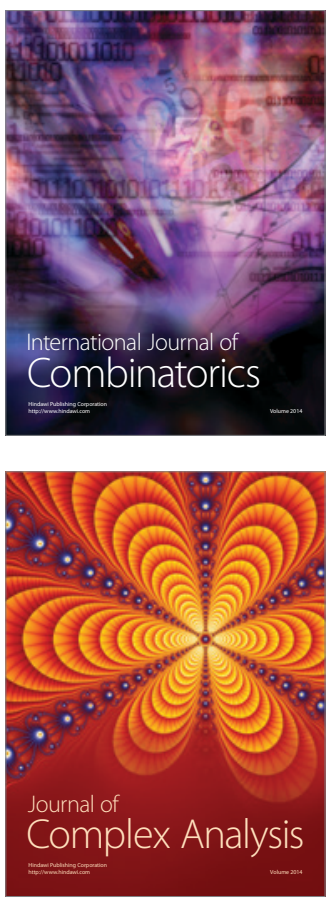

International Journal of

Mathematics and

Mathematical

Sciences
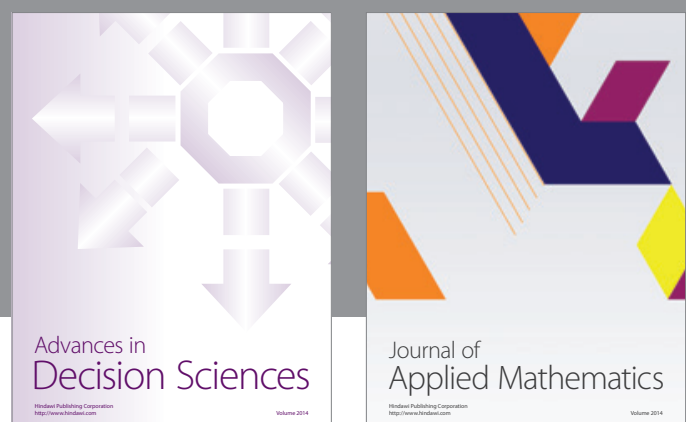

Journal of

Applied Mathematics
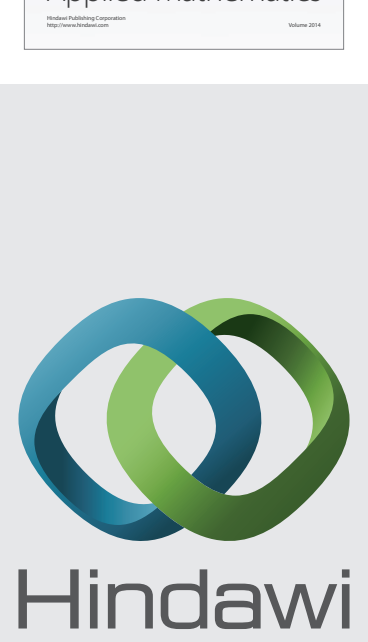

Submit your manuscripts at http://www.hindawi.com
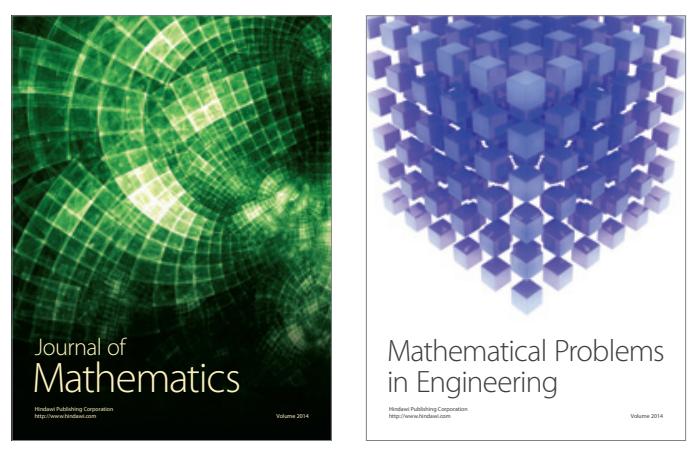

Mathematical Problems in Engineering
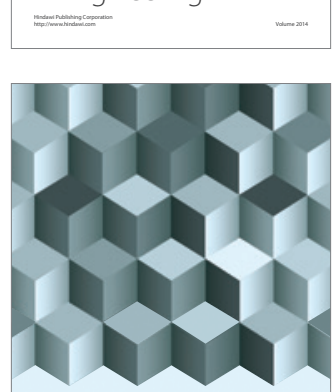

Journal of

Function Spaces
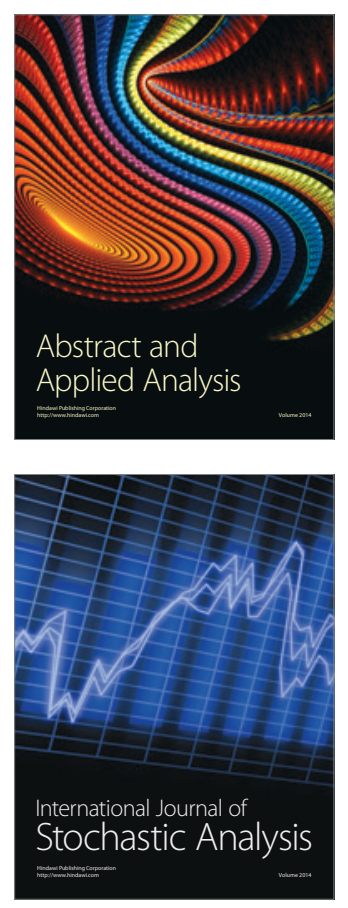

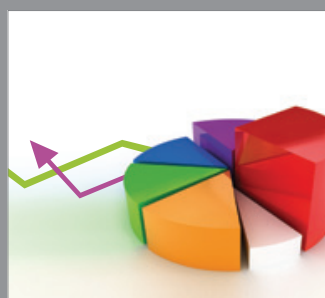

ournal of

Probability and Statistics

Promensencen
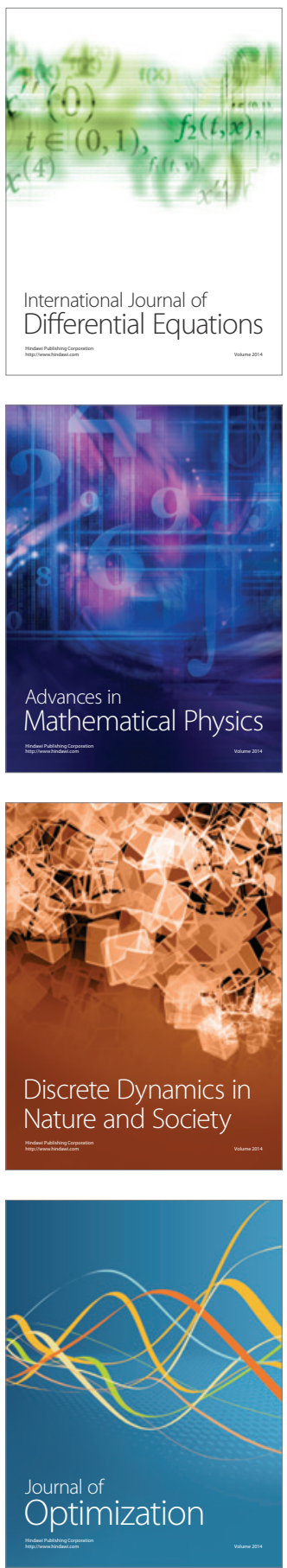